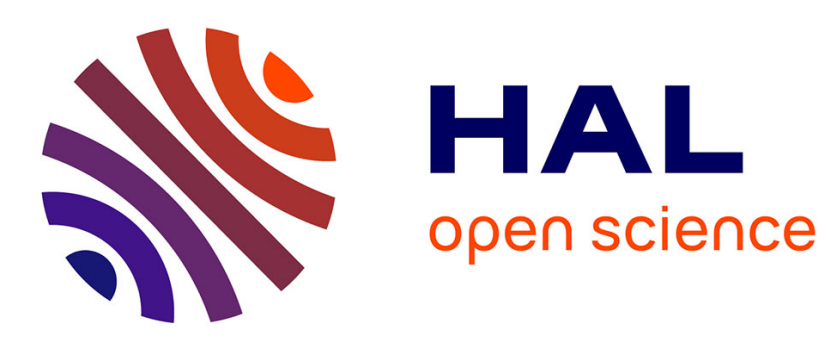

\title{
Regulation of the bimodal traffic
}

Nadir Farhi, Neila Bhouri, P Lotito

\section{To cite this version:}

Nadir Farhi, Neila Bhouri, P Lotito. Regulation of the bimodal traffic. CTS 2006 - 11th IFAC Symposium on Control in Transportation Systems, Aug 2006, Delft, Netherlands. pp.233-238, 10.3182/20060829-3-NL-2908.00041 . hal-01473659

\section{HAL Id: hal-01473659 \\ https://hal.science/hal-01473659}

Submitted on 22 Feb 2017

HAL is a multi-disciplinary open access archive for the deposit and dissemination of scientific research documents, whether they are published or not. The documents may come from teaching and research institutions in France or abroad, or from public or private research centers.
L'archive ouverte pluridisciplinaire HAL, est destinée au dépôt et à la diffusion de documents scientifiques de niveau recherche, publiés ou non, émanant des établissements d'enseignement et de recherche français ou étrangers, des laboratoires publics ou privés. 


\title{
REGULATION OF THE BIMODAL TRAFFIC
}

\author{
N. Farhi ${ }^{*}$, N. Bhouri ${ }^{* *} \&$ P. Lotito ${ }^{* *}$ \\ * INRIA - Rocquencourt, B.P. 105, 78153 Le Chesnay \\ Cedex France \\ ** INRETS - Arcueil, 2 av. Général Malleret-Joinville \\ 94114 Arcueil France \\ e-mail : Nadir.Farhi@inria.fr
}

\begin{abstract}
We consider an urban network with two traffic modes. We control the traffic lights in order to free the roads used by the public transport vehicles. To do this, we solve a flow assignement problem, from which we deduce an ideal distribution of private vehicles. Then using traffic lights, we design a regulator maintaining the car distributions in the roads around the ideal trajectory. This regulator is obtained by solving a linear quadratic problem. The result is a global feedback on the car numbers in the roads. We show the robustness of the obtained control. We compare the obtained results with those given by Bhouri and Lotito (2005).
\end{abstract}

Keywords: Traffic Modelling, Bimodal Traffic, Automatic, Traffic Assignment, Public Transportaion

\section{INTRODUCTION}

Real time traffic-responsive signal control (TRC) has the ability to improve traffic operations in urban areas when compared to traditional fixedtime control. The steadily growth of the mobility in urban areas offset however this improvement and roads are often congested. In recent years, an increasing awareness has been observed around the world for the potential contribution that public transport may have in the amelioration of the overall traffic conditions. For this reason, many measurements encouraging the use of the public transport means are achieved, among them the public transport priority (Diakaki et al.).

The TRC systems have been developed with consideration of the unimodal traffic. They have been extended later to give the priority at traffic lights to the public transport vehicles (PTV). These strategies take into account the PTV in a local treatment and not as a mode of traffic at the same level of the particular vehicles (PV). Nevertheless, we can quote the work of Bhouri and Lotito (2005) which consider two modes of traffic.

In this paper, we present a method to control traffic lights of a transportation system with two modes : PV and PTV. We want to control the traffic lights in order to free the roads used by the public transport vehicles.

We consider a network of roads with a traffic light controlling the output of each road. On this network, bus lines are given. The states are the car and the bus numbers in the roads, and the controls are the car outflows of the roads obtained by choosing the green/red phasing of the traffic lights.

We design a regulator maintaining the system around an ideal trajectory. This ideal trajectory is obtained by solving first a flow assignment giving the PV Wardrop equilibrium, from which we deduce the car numbers in the roads. Then by 
reducing the car numbers at the time and roads when/where are the PTV. The traffic assignment is obtained using the toolbox CiudadSim ${ }^{1}$ of Scilab $^{2}$. The regulator is obtained by solving a Linear Quadratic Problem.

We show the numerical results obtained on an academic example. We check, on this example, the controler robustness by introducing disturbances on the car numbers in the roads and on bus timetables.

We compare on the same example this methodology with the one given by Bhouri and Lotito (2005), where a LQ regulator is also computed but instead of regulating around an ideal trajectory, the standard quadratic criterium is modified in such a way to penalize the traffic on the roads used by the bus (the contoller thus obtained being modified to become admissible). We will call this method Modified LQ regulator (MLQR).

\section{MODELING}

A traffic road network is represented by a graph. The nodes of this graph correspond to the crossroads and the arcs to the roads or to inputs or outputs of cars in the system. The PTV follow fixed lines which are given by paths in the network. Let us take the academic example given

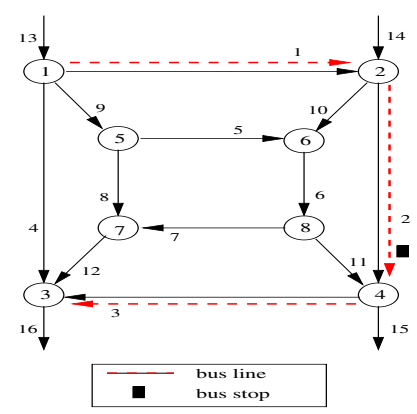

Fig. 1. Example

in Figure 1. It shows a graph corresponding to a small traffic network. This network has two inputs : (13) and (14), and two outputs : (15) and (16). The PTV follow only one line using the roads (1), (2) then (3), with a stop on the road (2). The traffic light on each crossroads is periodic and we use this period as the discetization time step.

\subsection{Dynamics of the $P V$}

The number of $\mathrm{PV}$ in a given road which is the state of the system is updated by adding the number of vehicles entering the road, and

\footnotetext{
1 http://www-rocq.inria.fr/metalau/ciudadsim

2 http://scilabsoft.inria.fr
}

deducting the number of vehicles leaving it. More precisely, we denote:

$\mathcal{N}:$ the set of nodes

$\mathcal{A}$ : the set of arcs

$\mathcal{I}_{c}$ : the set of the arcs entering the node $c$

$\mathcal{O}_{c}$ : the set of the arcs leaving the node $c$

$x_{k}^{a}$ : the number of PV circulating at the moment $k$ on the road a

$u_{k}^{a}$ : the outflow of the road a at the moment $k$ (controlled by network lights.)

$e_{k}^{a}$ : the number of $\mathrm{PV}$ entering the road $(a)$ at the moment $k+1$

$b_{a^{\prime} a}$ : the proportion of the $\mathrm{PV}$ which take the road $(a)$ among those which leave the road $\left(a^{\prime}\right)$

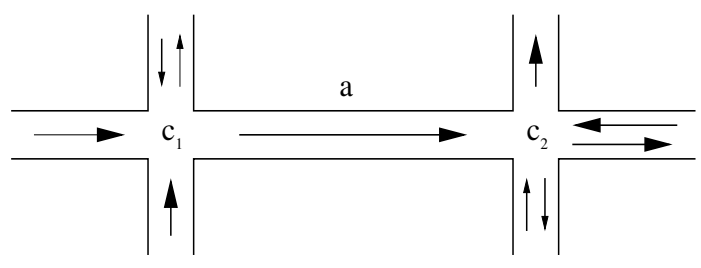

Fig. 2. Dynamics of the (PV)

Then we have

$$
x_{k+1}^{a}=x_{k}^{a}+\sum_{a^{\prime} \in \mathcal{I}_{c_{1}}} b_{a^{\prime} a} u_{k}^{a^{\prime}}+e_{k}^{a}-u_{k}^{a}
$$

This equation can be written like this

$$
x_{k+1}=x_{k}+B u_{k}+e_{k}
$$

where $B$ will be called the routing matrix.

\subsection{Dynamics of the PTV}

We consider stops on each bus line. A line is represented by a path and a word indicating the time spent in the roads. For example, the single line of the network on figure 1 is represented by the path $\left(\begin{array}{lll}1 & 2 & 3\end{array}\right)$ and by the word 121 which refers to a stop of 1 unit of time on the arc 2 and to 1 unit of travel time on each road.

Let's denote:

$y_{k}^{h a}:$ the number of PTV of the line $h$ on the road $a$ at time $k$

$\tau$ : the estimated time spent on a road where there is a stop (In the following, $\tau$ will be equal to 2 , and we will suppose that on all the other roads, the bus spends one unit of time).

$a, a^{\prime}$ : two successive arcs of a line.

The PTV dynamics can be written:

$y_{k+1}^{h a^{\prime}}= \begin{cases}y_{k-\tau}^{h a}, \tau \in \mathbb{N}^{*} & \text { if there is a stop on } a \\ y_{k}^{h a} & \text { otherwise. }\end{cases}$ 


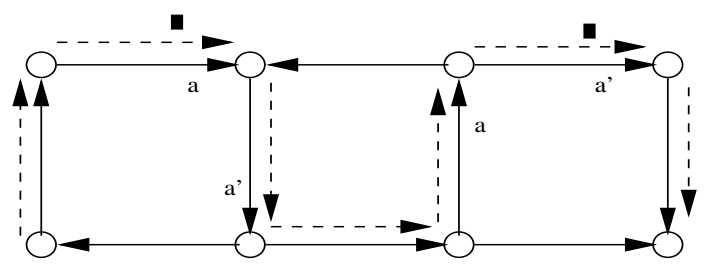

Fig. 3. Dynamics of the PTV

\subsection{How to obtain the ideal trajectory}

We compute an ideal distribution of $\mathrm{PV}$ on the roads, around which a regulator will be designed in the next section. One way of calculating it, is first to solve a traffic assignment problem which determine the car numbers in the roads and the output flows in the absence of priority given to PTV. Then, the ideal distribution of cars on a road used by PTV at a given time is obtained by reducing the number of $\mathrm{PV}$ on this road at this time.

The flow assignment problem consists of determining the flows $f$ on the $\operatorname{arcs} a \in \mathcal{A}$, knowing that journey times $t_{a}$ of $\operatorname{arcs} a$ are function of the flows $f_{a}$. A network equilibrium gives us these flows.

Wardrop Equilibrium. Each user minimizes its time spent in the network. Therefore at Wardrop equilibrium (user equilibrium), for each pair of nodes $(p, q)$ of traffic demand from $p$ towards $q$, the time spent on all the used routes from $p$ to $q$ are the same, and they are less than the time spent on any unused route from $p$ to $q$. Let's note :

- $\mathcal{D}$ : The set of pairs of traffic demand : origindestination

- $\mathcal{R}_{p q}$ : The set of paths from the origin $p$ to the destination $q$

- $\mathcal{R}^{a}$ : The set of all the paths which include the arc a

- $d_{p q}$ : The demand from $p$ to $q$

- $f_{r}$ : The flow on a path $r \in \mathcal{R}_{p q}$

- $f^{a}$ : The flow on the arc $a$

- $t_{r}$ : The journey time on a path $r \in \mathcal{R}_{p q}$

- $t_{p q}^{*}$ : The shortest time of journey from $p$ to $q$

- $t^{a}\left(f^{a}\right)$ : The journey time on the arc $a$, function of the flow $f^{a}$ on this arc

A vector $f$ whose components $f_{r}$ represent the flows on the paths $r$ is a Wradrop Equilibrium if:

$$
\begin{gathered}
f_{r}\left(t_{r}-t_{p q}^{*}\right)=0, \quad t_{r}-t_{p q}^{*} \geq 0, \quad f_{r} \geq 0 \\
t^{*} \geq 0, \quad \sum_{r \in R_{p q}} f_{r}=d_{p q}, \quad \forall r \in R_{p q}, \forall p, q \in D
\end{gathered}
$$

The variational formulation of the equilibrium :

$$
\left\{\begin{array}{c}
\min _{f} \sum_{a} \int_{0}^{f^{a}} t_{a}(s) d s, f^{a}=\sum_{r \in R^{a}} f_{r} \\
\sum_{r \in R_{p q}} f_{r}=d_{p q}, \quad f \geq 0
\end{array}\right.
$$

The problem (3) being static, we consider several stationnary regimes $\mathcal{S}=\left\{S_{1}, \cdots, S_{m}\right\}$ and we solve an assignment problem for each regime. The $S_{i}$ are time sections : $S_{1}=\left\{0, \cdots, i_{1}\right\}, S_{2}=\left\{i_{1}+\right.$ $\left.1, \cdots, i_{2}\right\}, \cdots, S_{m}=\left\{i_{m-1}+1, \cdots, T\right\}$.

The numerical solving of these problems (3) are done thanks to the CiudadSim toolbox of Scilab. We obtain :

$f_{S}^{*}$ : the optimal solution of the problem (3) on a time section $S \in \mathcal{S}$;

$t_{S}^{a}$ : the journey time on the arc $a \in \mathcal{A}$ during the time section $S$.

\subsection{Resolution of the linear quadratic problem}

To determine a regulator stabilizing the system around the ideal trajectory we solve a Linear Quadratic (LQ) problem whose dynamics is given by the equation (1), and whose criterion is the quadratic distance to the ideal trajectory.

The problem to solve is :

$$
\left\{\begin{array}{c}
\min _{u} \sum_{k=0}^{T-1}\left\{\left(x_{k}-\bar{x}_{k}(y)\right)^{\prime} Q\left(x_{k}-\bar{x}_{k}(y)\right)\right. \\
\left.+\left(u_{k}-\bar{u}_{k}\right)^{\prime} R\left(u_{k}-\bar{u}_{k}\right)\right\} \\
x_{k+1}=x_{k}+B x_{k}+e_{k}
\end{array}\right.
$$

where :

- $Q$ and $R$ are weighting matrices that we take diagonal $Q=\lambda_{1} I_{1}, R=\lambda_{2} I_{2},\left(\lambda_{1}, \lambda_{2}\right) \in \mathbb{R}_{+}^{2}$ and $I_{1}$ and $I_{2}$ are the corresponding identity matrices,

- the ideal flow $\bar{u}$ is given by the assignment problem :

$$
\bar{u}_{k}=f_{S}^{*}, \quad \forall S \in \mathcal{S} \text { et } \forall k \in S
$$

- the car numbers in the roads $\widetilde{x}$ are given using the Little formula :

$$
\widetilde{x}_{k}^{a}=f_{S}^{* a} \cdot t_{S}^{a}\left(f_{S}^{* a}\right), \quad \forall a \in \mathcal{A}, \forall S \in \mathcal{S} \text { et } \forall k \in S
$$

- the ideal car numbers in the roads are obtained mainly by dividing these quantities by the number of PTV on the road at this epoch.

$\bar{x}_{k}(y)=\frac{\beta}{1+\sum_{h} y_{k}^{h}} \cdot \widetilde{x}_{k}, \quad \beta:$ a positive parameter

We solve the LQ problem (4) by integrating the corresponding Riccati equation. Then the global feedback is :

$$
u_{k}-\bar{u}_{k}=K_{k}\left(x_{k}-\bar{x}_{k}\right)+L_{k},
$$

where $K_{k}$ and $L_{k}$ are the gains deduced from the Riccati equation. 


\section{NUMERICAL SOLVING}

Let us take the example of the figure (1) which represents a network of 16 roads including 2 entries and 2 exits, one bus line $(1,2,3)$ with a stop on road 2 and 4 traffic demand pairs : $\{1 \rightarrow 3,1 \rightarrow 4,2 \rightarrow 3,2 \rightarrow 4\}$. The 4 demands, given in Table 1, are supposed to be the same, and equal to 100 vehicles/time-unit for all the time sections.

To check if the obtained PV traffic is reduced on the roads where are the PTV, we take three time sections with different time-tables given by Table 2 .

\begin{tabular}{||c||c|c|c||}
\hline section time & $S_{1}$ & $S_{2}$ & $S_{3}$ \\
\hline \hline Time & 0 to 20 & 21 to 70 & 71 to 100 \\
\hline Orig.-Dest. & all pairs & all pairs & all pairs \\
\hline PV Flow & 100 & 100 & 100 \\
\hline
\end{tabular}

Table 1. PV Flows

\begin{tabular}{||c||c|c|c||}
\hline Time section & $S_{1}$ & $S_{2}$ & $S_{3}$ \\
\hline \hline Time & 0 to 20 & 21 to 70 & 71 to 100 \\
\hline PTV Flow & 1 & $1 / 3$ & $1 / 2$ \\
\hline
\end{tabular}

Table 2. PTV Timetables

Figure 4 gives the PV flow obtained by the Wardrop Equilibrium. The thickness of an arc on this figure is proportional to the flow on the road. We point out that this assignment does not take into account the PTV flows.

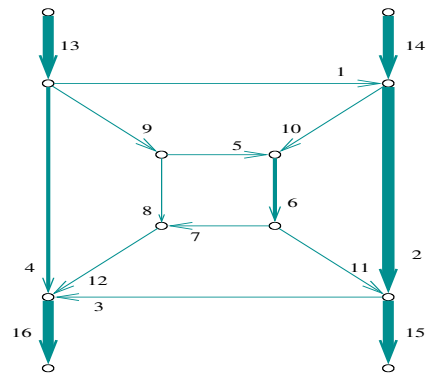

Fig. 4. The flow assignment

We solve the regulator problem (4) and we simulate the closed loop system (with the regulator) in different cases. The results are shown in Figure 5.

On this figure we have a table of 3 lines and 3 columns. Each line of this table corresponds to one of the three $\operatorname{arcs} 1,2$, and 10, and each column corresponds to one simulation.

Column 1 corresponds to simulation without disturbance, column 2 corresponds to simulation with a disturbance on the number of $\mathrm{PV}$ in a road ( $x$ disturbance), and column 3 corresponds to simulation with a disturbance on the PTV timetable ( $y$ disturbance).
On these curves, the $\mathrm{x}$-axis represents time, and the $y$-axis represents number of vehicles. On each of the 9 subfigures, there are two trajectories : the car numbers trajectory on the top, and the bus numbers one on the bottom with an adapted scaling.

On column 1 of the table, we can see that the car traffic follows perfectly the bus one on the roads 1 and 2 . The road 10 is not used by the bus but it is influenced by roads 1 and 2 (see the graph of the Figure 1).

To measure the robustness of the regulator, we introduce two kinds of disturbances : one on car numbers and the other one on the bus numbers.

Firstly, we disturb the car numbers by applying the following dynamics :

$$
x_{k+1}=(1+w) x_{k}+B u_{k}+e_{k}
$$

where $w \hookrightarrow \mathcal{N}(0,1 / 2)$.

This disturbance is about $50 \%$ of the PV numbers. We represent the results on the column 2 of Figure 5.As we can see on these curves, the number of (PV) still follows that of (PTV), that is to say that they remain complementary.

Secondly, we disturb the bus numbers by replacing $y_{k}^{h}$ by $(1+w) y_{k}^{h}$ during the simulation. This disturbance is about $50 \%$ of the PTV number. The results of the simulation are given on the column 3 of Figure 5 . The control remains correct.

\section{COMPARISON WITH THE MODIFIED LQ REGULATOR (MLQR)}

In this section, we try to compare our work with that done by Bhouri and Lotito (2005). Their formulation consists of solving the following problem:

$$
\left\{\begin{array}{l}
\min _{u} \sum_{k=0}^{+\infty}\left[x_{k}^{\prime} Q_{1} x_{k}+x_{k}^{\prime} Q_{2} y_{k}^{\prime}+u_{k}^{\prime} R u_{k}\right] \\
\quad x_{k+1}=x_{k}+B u_{k}
\end{array}\right.
$$

The dynamics of the problem 5 is the same as that of the problem 4 . However, the criteria of the two problems differ. The advantage of the criterion of the problem 5 is that we don't need to compute an ideal trajectory. So we can avoid resolving the flow assignment problem. But its drawback is that it gives a non admissible policy $((x, u)$ is admissible if $x \geq 0$ and $0 \leq u \leq u^{+}, u^{+}$given $)$. Therefore we have to derive an admissible solution from the MLQR solution.

For that let us define the two matrices $B^{+}$and $B^{-}$as follows :

$$
\left\{\begin{array}{l}
B_{i j}^{+}=\max \left\{0, B_{i j}\right\} \forall i, j \\
B_{i j}^{-}=\max \left\{0,-B_{i j}\right\} \forall i, j
\end{array}\right.
$$




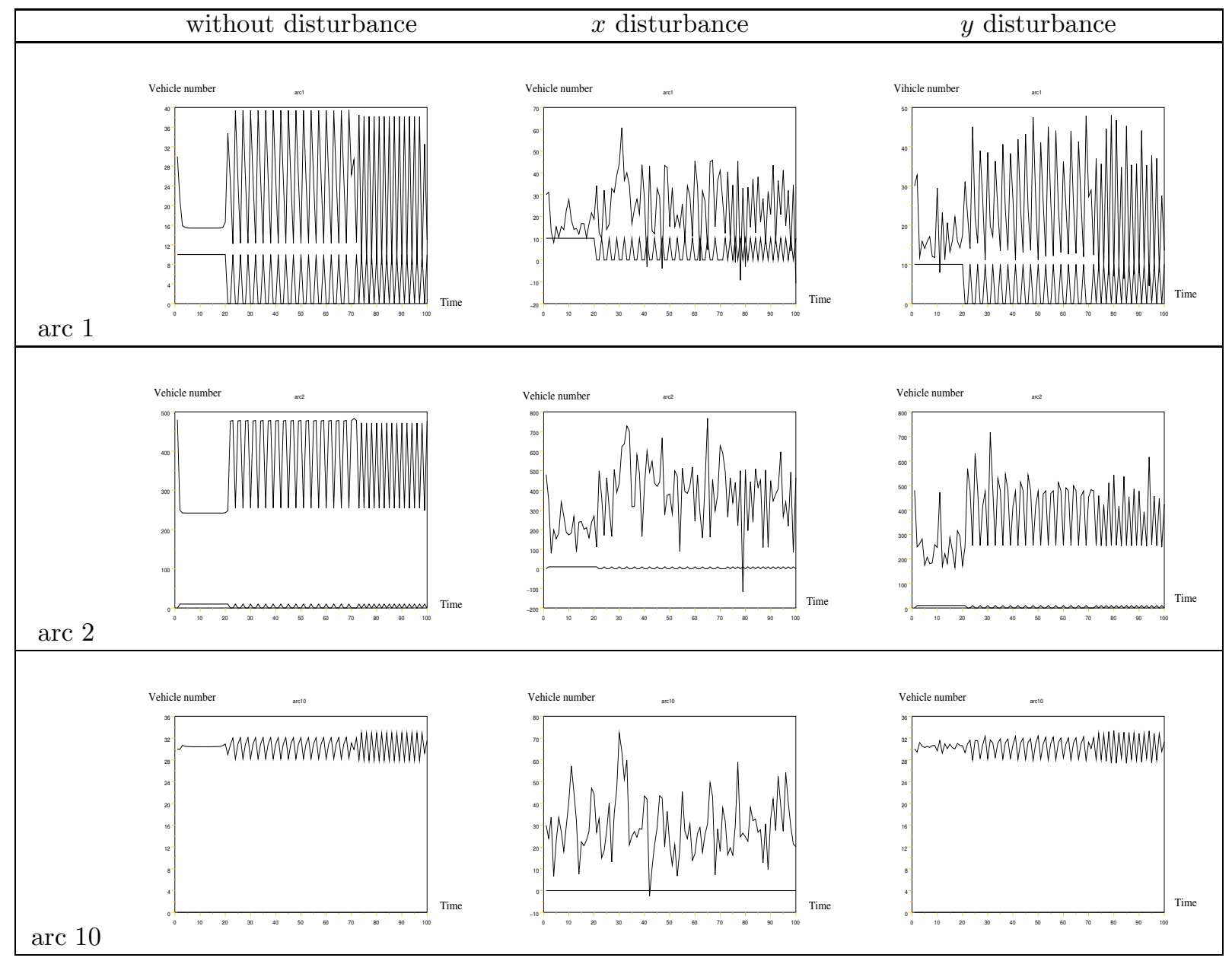

Fig. 5. Results of simulations: without disturbance, with a disturbance of the number of PV (x disturbance), and with a disturbance of the number of PTV (y disturbance).

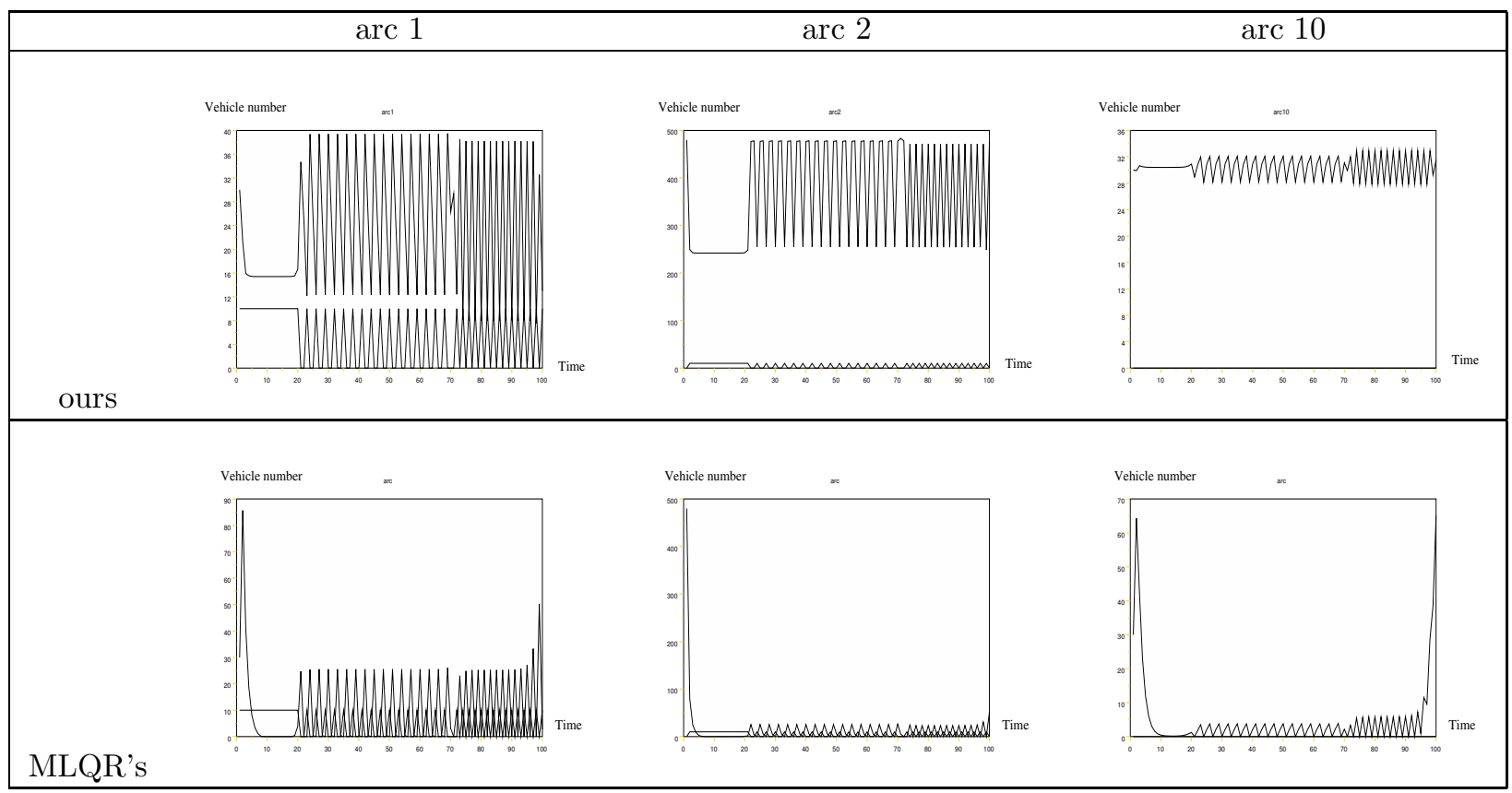

Fig. 6. Comparison of two approaches: our approach (ours) and the MLQR (MLQR's) 
Since it is supposed that there is one control by road, the matrix $B^{-}$is a permutation matrix and its inverse transforms the positive cone to the positive cone then it is easy to obtain an admissible feedback from a not admissible one. At time $k$, taking as road inflows the outflows at time $k-1$, the outflows at time $k$ are obtained by inverting the matrix $B^{-}$. More precisely, let's denote $u_{k}$ the control given by the MLQR feedback at the moment $k, \widehat{u}_{k}$ give the control to be applied : $\left\{\begin{array}{l}x_{0} \geq 0 \\ v_{0}=0\end{array} \quad\right.$ given

$\left\{\begin{array}{l}x_{k}=x_{k-1}+v_{k-1}-B^{-} \widehat{u}_{k-1} \\ v_{k}=B^{+} \widehat{u}_{k-1} \\ u_{k}=\text { the feedback result at the moment } k \\ \widehat{u}_{k}=\max \left\{0, \min \left\{u_{k}, u^{+},\left(B^{-}\right)^{-1}\left(x_{k}+v_{k}\right)\right\}\right\}\end{array}\right.$

On Figure 6, we give the results of a simulation without disturbance. The Table of this Figure contains two lines, the first correponds to the formulation presented in this paper, and the second corresponds to the MLQR formulation.

Each column of this table corresponds to one of the three $\operatorname{arcs} 1,2$ and 10. Surprisingly, as we can see, the MLQR formulation gives the same kind of qualitative stationary regime but have larger initial excursions. Let us remark that in MLQR method, we have not to solve the traffic assignment problem which needs a lot of non reliable data on the system. Indeed in general we don't know the origine destination demands necessary to compute the Wardrop equilibrium.

\section{CONCLUSION}

We have presented here a way to manage the traffic in an urban network with two modes (cars and buses). Our method is based on the traffic light control of the car flows in order that buses be in time.

Let's remark that we have only studied the simple light control phase case : on each road there is an independent traffic light which controls the access to the crossroad. If this light is green, the PV being on this road can choose any other road to leave. With this assumption the routing matrix $B$ is full rank. This guarantees the commandability of the system. However, in practice, we often need to use more complicated phases.

In this simple case, the linear quadratic problem around an ideal trajectory gives a robust control. It needs the solution of a traffic assignment problem for which it is difficult to obtain reliable data. An alternative approach avoid this difficulty and seems to obtain the same qualitative results.

The academic example used here is encouraging but too simple to conclude to the robustness of this approach. Study on more realistic networks would be useful.

\section{REFERENCES}

Bhouri Neila \& Lotito Pablo. (2005). An intermodal traffic control strategy for private vehicle and public tranport. 10th Euro Working Group on Transportation Poznan Poland.

Braban C. and Boillot F. (2003). Les systèmes temps réel de commande de feux en milieu urbain. Les collections de l'INRETS.

Chen W., Jarjees G. \& Drane C. (1998). A new approach for bus priority at signalised intersections. 19th ARRB Transport Research LTD Conference, Sydney Australia.

CiudadSim http://www-rocq.inria.fr/metalau/ciudadsim

Diakaki Christiana, V. Dinopoulou \& Papageorgiou Markos. Public transport priority. SMART NETS project.

Diakaki Christiana, Papageorgiou Markos, \& Aboudolas Kostas. (2002). A multivariable regulator approach to traffic-responsive networkwide signal control. Control Engineering Practice, 10, 183-195.

Scabardonis A. (2000) Control strategy for transit priority. TRR vol. 1727, 00-1584, 20-26.

Scemama Gérard. (December 1994). La conception de plan de feux : une modélisation par la programmation sous contraintes. Recherche Transports Sécurité. No 45.

Scilab http://scilabsoft.inria.fr

Wardrop J. G., B.A. (1952). Some theoritical aspects of road traffic research. Proc. Institute of civil Engineering, Part II 1, 325-378.

Yagar S. (1991). Consise encyclopedia of Traffic and Transportation systems. Pergamon Press 